\title{
Final Technical Report for DOE Grant DE-FG02-03ER15474
}

\author{
Wilfred T. Tysoe \\ Department of Chemistry and Biochemistry, and Laboratory for Surface Studies \\ University of Wisconsin-Milwaukee
}

\section{Catalysis Science: Molecular Level Design of Chiral Heterogeneous Catalysts}

\section{Overall Progress}

The central philosophy of this project is to examine the chemisorptive enantioselectivity of a range of chiral modifiers to identify the molecular-level requirements for the successful operation of a chiral modifier. Work has focused on two general areas. The first area examines those modifiers that are expected to act as chiral templates, that is, to operate in a collective fashion to define chiral ensembles at the surface. The second area investigates the properties of so-called "one-to-one" modifiers where enantioselectivity is imparted by a direct interaction between a chiral modifier and a prochiral reactant.

Previous work has shown that the adsorption of R- or S-2-butanol on a Pd(111) surface can be used to create an enantioselective, templated chiral surface. The enantioselectivity was probed by the adsorption of R- or S-propylene oxide, where coverages were measured both using temperature-programmed desorption (TPD) and infrared spectroscopy. Since propylene oxide adsorbs and desorbs reversibly on $\operatorname{Pd}(111)$, this provides an ideal probe molecule. Enantioselective adsorption of the propylene oxides is observed over a narrow range of the 2 -butanol coverage near $\theta_{\text {but }}=1 / 2$ monolayer. The enantioselective ratio for adsorption of propylene oxide on the templated surface is defined as:

$$
E R_{2-\text { butanol }}=\frac{\theta_{R-\text { propyleneOxide }}}{\theta_{S-\text { PropyleneOxide }}}
$$

and can reach values as high as $E R_{2 \text {-butanol }}=2$ on $\operatorname{Pd}(111)$. Work in this area has also been performed in collaboration with the Zaera group on $\operatorname{Pt}(111)$ [1], where enantioselectivity was also found.

2-butanol reacts on heating to form methyl-ethyl ketone via a $\beta$-hydride elimination reaction resulting in the destruction of the chiral center with a concomitant decrease in the value of $E R_{2 \text {-butanol }}$ to unity, as expected $[2,3]$. In order to provide a more stable templating species, we have made use of R- and S-2-methyl butanoic acid as the chiral template on $\operatorname{Pd}(111)$ since this has the same chiral center as 2-butanol, but is more strongly bonded to the surface through a carboxylate group. However, for this templating agent there is no enantioselective adsorption of propylene oxide $\left(E R_{\text {2-methyl butanoic acid }}=1\right)$. This may arise from the fact that the chiral butyl group is now located farther away from the surface than in the case of the 2-butanol templates and can therefore rotate more 
freely, averaging out any asymmetry, thus reducing its chirality. However, other explanations are possible and these are currently being investigated. However, in support of this proposal, functionalizing the butyl group with an amine (to form 2-amino butanoic acid) restores enantioselectivity for the adsorption of propylene oxide, $E R_{2 \text {-amino butanoic acid }}$ $=1.75 \pm 0.06$. This suggests that an effective chiral template requires the chiral center to

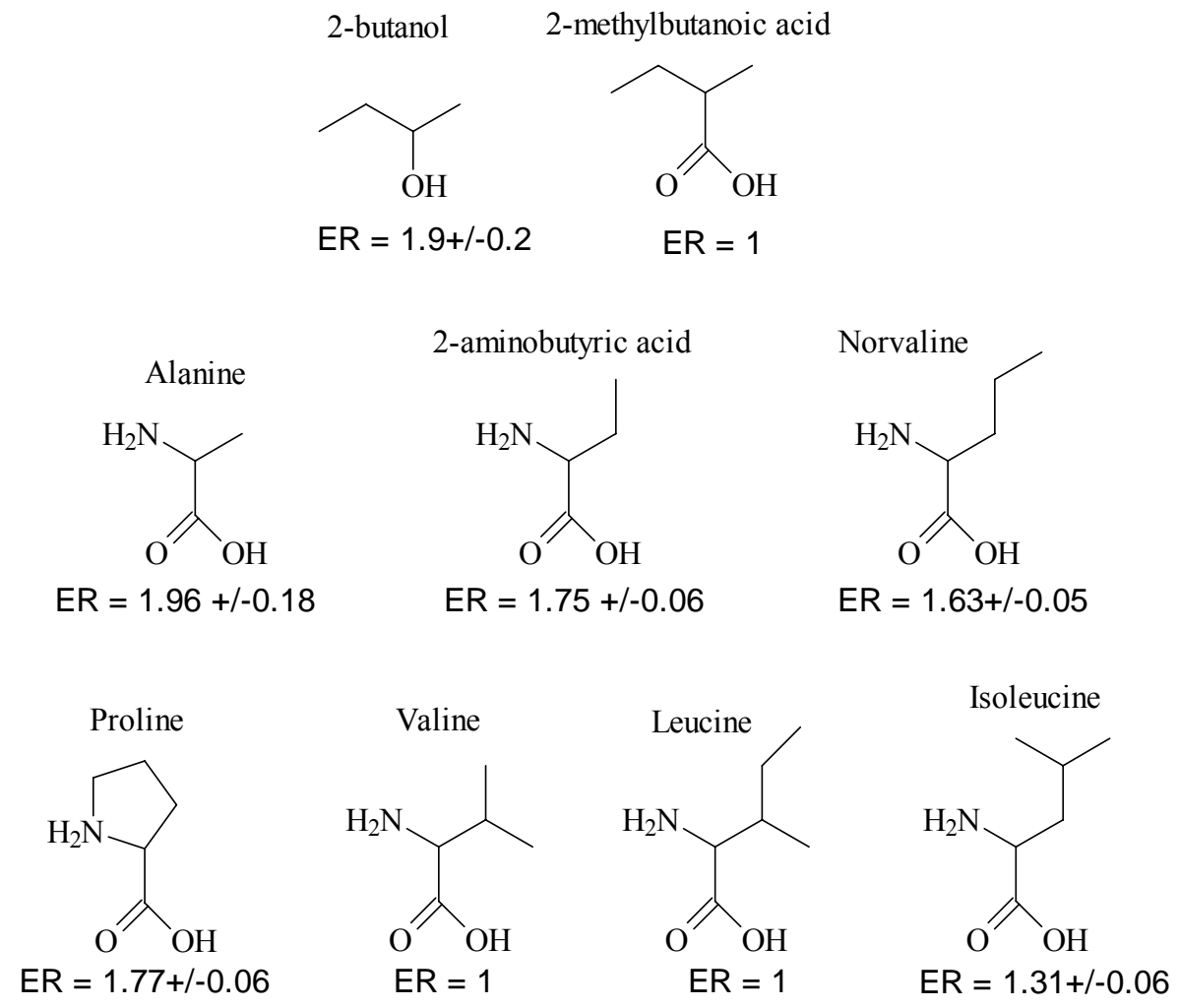

Figure 1: List of template molecules and their associated enantioselectivity ratios.

be anchored to the surface to prevent it from rotating freely in accord with the above proposal. The ER value was therefore measured using a number of simple amino acids as shown in Fig. 1 using propylene oxide as a probe. In these cases, the templated surface was prepared using either the R- or S-aminoacid, then exposed to R-propylene oxide, and the propylene oxide coverage measured. Since the aminoacids are stable on the surface at the temperature at which propylene oxide desorbs, the surface was then thermally depleted of R-propylene oxide and then exposed to S-propylene oxide to measure $E R$. Once again, in all cases the enantioselectivity ratio varies with adsorbate coverage reaching a maximum at approximately $50 \%$ of saturation in accord with the notion that these molecules act as templates $[4,5]$.

The maximum enantioselectivity ratios for each of these templates are plotted in Fig. 2 versus the number of carbon atoms in the R-group in the amino acid

$\mathrm{C}^{*} \mathrm{H}(\mathrm{COOH})\left(\mathrm{NH}_{2}\right) \mathrm{R}$, where $\mathrm{C}^{*}$ is the chiral carbon. This emphasizes that the $E R$ value, using the identical propylene oxide probe, decreases monotonically with increasing $n$ - 
alkyl carbon chain length, as illustrated by alanine $\left(\mathrm{R}=\mathrm{CH}_{3}\right)$, 2-aminobutyric acid $\left(\mathrm{R}=\mathrm{C}_{2} \mathrm{H}_{5}\right)$, and norvaline $\left(\mathrm{R}=\mathrm{C}_{3} \mathrm{H}_{5}\right)$. Since these molecules are proposed to act by defining a "chiral pocket" at the surface, this result implies that the size of the chiral pocket increases only slightly with increasing chain length of the R-group. Since the change in ER ratio is relatively modest as the chain length changes from $C_{1}$ to $C_{3}$, this suggests that the size of the chiral pocket is defined primarily by the $\alpha$-carbon and that the remainder of the carbon atoms are oriented away from the surface. This postulate is also in accord with the observation that branched alky groups (valine and leucine) have

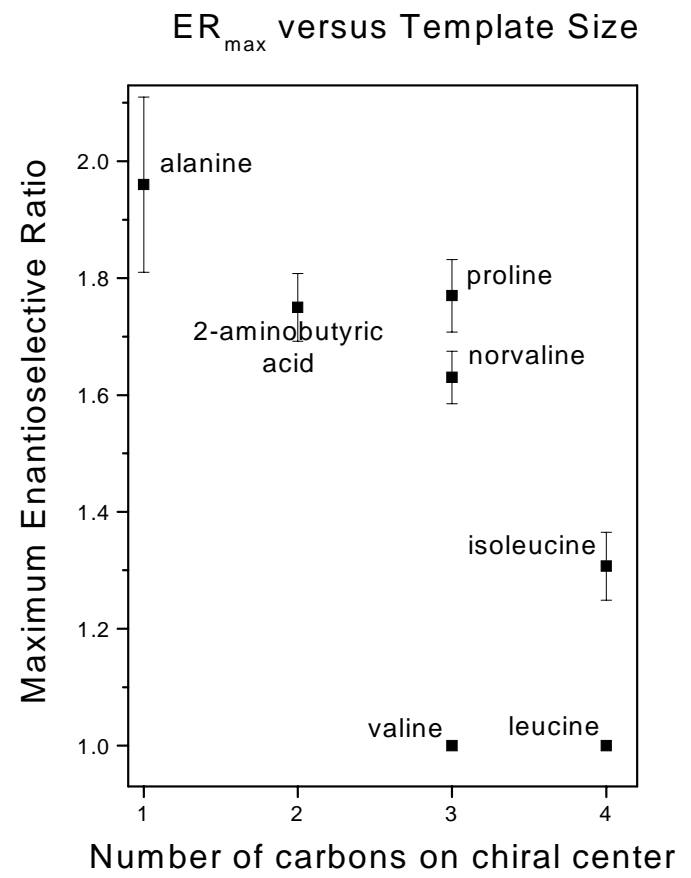

Figure 2: Plot of the maximum $E R$ ratio for a range of amino acids, versus the number of carbon atoms in the R-group.

an $E R$ ratio of unity. The presence of branched chains will lead to extremely large pockets that are no longer chiral. The origin of the relatively large ER ratio of proline is not clear and the explanation of this effect will rely on more detailed structural measurements.

In view of these results, we have explored the surface chemistry and structure of the aminoacids on $\operatorname{Pd}(111)$. The goal has been to measure the structure of aminoacids on $\operatorname{Pd}(111)$ using low-energy electron diffraction (LEED) intensity versus electron energy (I/E) measurements. We have developed, in collaboration with Professor Saldin from the Physics Department at UWM, a method for measuring the structures of disordered overlayers on single-crystal metal surfaces from the way in which the $\mathrm{I} / \mathrm{E}$ curves of the substrate $(1 \times 1)$ Bragg spots are modified by the presence of an adsorbate [6]. It has been demonstrated that this effect is approximately two orders of magnitude larger than the conventionally used diffuse background scattering. In order to determine the preferred 
binding site for the carboxylate group on $\operatorname{Pd}(111)$, the structures of formate and acetate species were measured on Pd(111) using LEED I/E curves. Since these do not form ordered overlayers, they are ideally suited to structural measurement using the method described above. In parallel with these measurements, we have collected reflectionabsorption infrared reflection spectra (RAIRS) of these species on $\operatorname{Pd}(111)$ to obtain infrared fingerprints of the carboxylate group and to establish the conditions under which the carboxylate is present on the surface and identify any possible co-adsorbed species (e.g., CO).

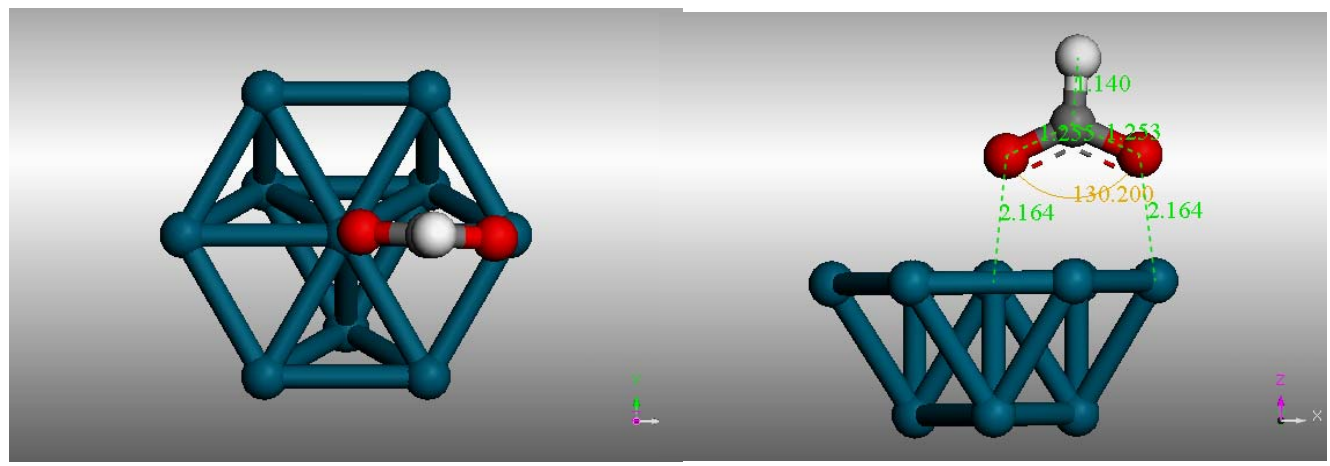

Figure 3: Schematic depiction of the structure of formate species on $\operatorname{Pd}(111)$

Fig. 3 depicts the resulting structure of formate species on $\mathrm{Pd}(111)$ indicating that the formate oxygen atoms are located approximately above surface palladium atoms. The structure of the acetate species is identical with the methyl group oriented normal to the surface. RAIRS results reveal the formation of some $\mathrm{CO}$ from formate decomposition when the initial formic acid coverage is below saturation, but no $\mathrm{CO}$ when formate species are formed from formic acid coverages greater than saturation (i.e., when multilayers are formed).

Determining the structure of aminoacids on Pd(111) using LEED turned out to be much more complicated since these decompose extremely rapidly in the electron beam. We have therefore collaborated with Professor Hirschmugl in the Department of Physics at the University of Wisconsin-Milwaukee who has built a LEED system in which a full I/E curve can be collected at a sufficiently low incident current that less than one electron has

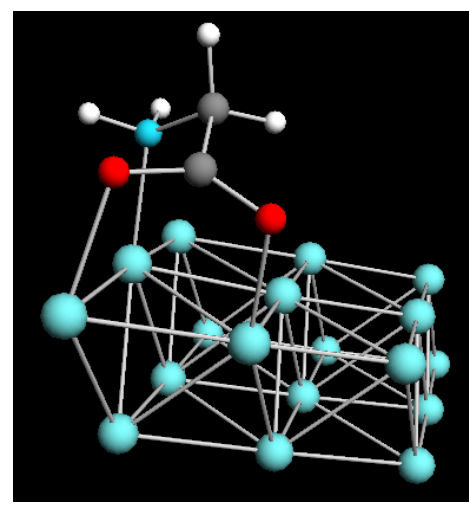

Figure 4: Calculated structure of glycine on $\operatorname{Pd}(111)$ (Sholl group) 
interacted with each surface adsorbate, thereby completely eliminating electron beam decomposition effects. We have successfully collected I/E curves for glycine, alanine and 1-(1-naphthyl)-ethylamine (see below) and these data are currently being analyzed to determine the adsorbate structures. In order to assist with the analysis of the LEED I/E structures, the structures of aminoacids on $\operatorname{Pd}(111)$ have been calculated using density functional theory by the Sholl group and an example structure is shown in Fig. 4.

The surface chemistry of small aminoacids has also been explored on Pd(111) using a combination of X-ray photoelectron spectroscopy (XPS) and temperature-programmed desorption (TPD). It is found that the aminoacids adsorbs predominantly in their zwitterionic form on $\operatorname{Pd}(111)$ and are stable to $\sim 300 \mathrm{~K}$. Thermal decomposition occurs above this temperature predominantly by scission of the carboxylate-carbon bonds as depicted in Fig. 5. The resulting carboxylate moiety desorbs as carbon monoxide or carbon dioxide [7,8]. The nitrogen-containing fragment either desorbs as an amine or undergoes additional reaction at the surface to finally form $\mathrm{HCN}$. This reaction pathway is summarized in Fig. 5.

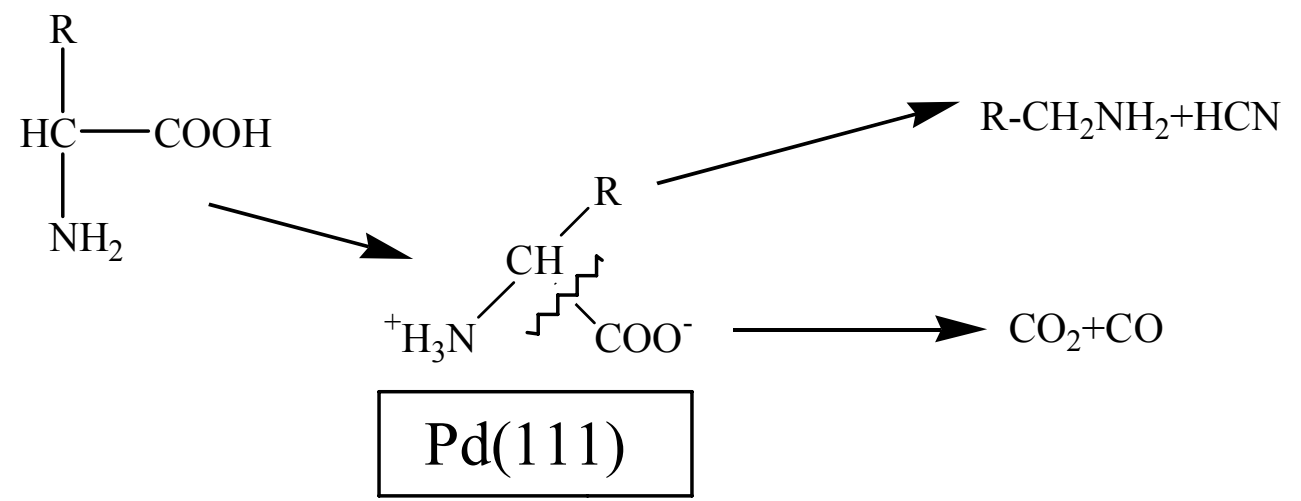

Figure 5: Summary of the reaction pathways for aminoacids on $\operatorname{Pd}(111)$

The most widely studied enantioselective heterogeneous catalyst is based on the templating of $\mathrm{Pt}$ with cinchonidine and provides an example of a putative one-to-one modifier. Unfortunately, this is rather difficult to introduce into ultrahigh vacuum. We have therefore focused on a simpler analog, 1-(1-naphthyl)-ethylamine, which has been shown to be a chiral modifier and is analogous to cinchonidine where the naphthyl ring anchors the molecule to the surface and the ethylamine group provides the chiral center.

The TPD spectra for S-1-(1-naphthyl)-ethylamine adsorbed on $\operatorname{Pd}(111)$ are displayed in Fig. 6 where hydrogen $(2 \mathrm{amu})$ and $\mathrm{HCN}(27 \mathrm{amu})$ are evolved. These spectra are similar to those found for 1-(1-naphthyl)-ethylamine on $\mathrm{Pt}(111)$ [9] suggesting that we have been able to adsorb the pure modifier onto the surface. The corresponding infrared spectra are displayed in Fig. 7 (left panel) where the $775 \mathrm{~cm}^{-1}$ feature is assigned to an $\mathrm{NH}_{2}$ wag, the $797 \mathrm{~cm}^{-1}$ mode to a $\mathrm{CH}$ ring bending mode and the $1371 \mathrm{~cm}^{-1}$ peak to a combined $\mathrm{CH}_{3}$ deformation $+\mathrm{C}-\mathrm{C}$ rings stretching mode. The relative intensities of these features varies substantially with coverage indicating a change in adsorbate orientation with coverage. 
This is confirmed by the CO titration data in Fig. 7 (right panel) showing the suppression
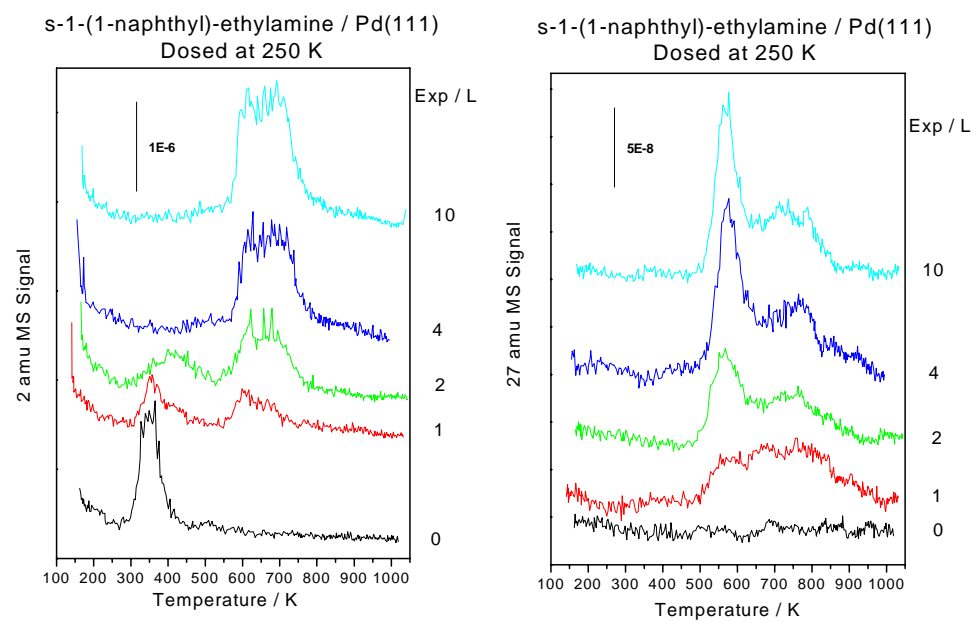

of $\mathrm{CO}$ adsorption, corresponding to saturation of the monolayer. The NEA coverage has

Figure 6: 2 and 27-amu temperature-programmed desorption spectra of S1-(1-naphthyl)-ethylamine on $\operatorname{Pd}(111)$

also been measured using XPS and the resulting uptake curve is shown in Fig. 8 (left panel). This suggests that NEA saturates at a coverage of $\sim 0.08$ monolayers and a depiction of the flat-lying geometry of NEA is also shown in Fig 8. The close-packed structure yields a saturation coverage in good agreement with that measured experimentally.

Experiments were carried out to measure the ER ratio of propylene oxide on 1-(1naphthyl)-ethylamine-modified $\operatorname{Pd}(111)$ and find the $\mathrm{ER}_{1 \text {-(1-naphthyl)-ethylamine }}=1$ at all 1-(1-
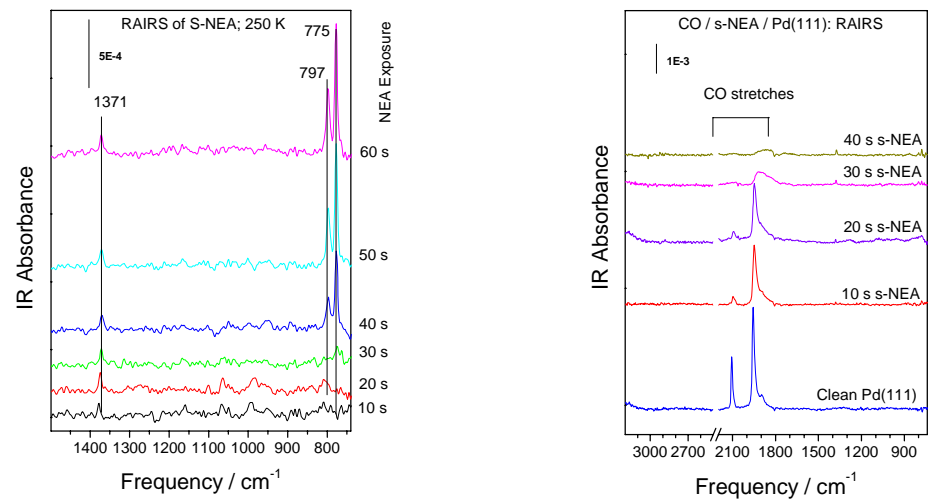

Figure 7: Reflection-absorption infrared spectra (RAIRS) of S-1-(1naphthyl)-ethylamine on $\operatorname{Pd}(111)$ as a function of exposure (left panel) and the RAIRS of CO adsorbed on S-1-(1-naphthyl)-ethylamine-covered $\operatorname{Pd}(111)$. 
naphthyl)-ethylamine coverages. It is likely, however, that one-to-one modification in

this case requires a more specific (hydrogen-bonding) interaction between the modifier and probe. We have therefore used R- and S-2-butanol as a probe for enantioselectivity on S-1-(1-naphthyl)-ethylamine-covered $\mathrm{Pd}(111)$. In this case, 2-butanol does not desorb molecularly, so that these experiments were carried out using a fresh 1-(1-naphthyl)ethylamine-modified $\operatorname{Pd}(111)$ surface for each experiment. The 1-(1-naphthyl)ethylamine was dosed from a Knudsen source with the 1-(1-naphthyl)-ethylamine thermostatted in an ice-water mixture so that exposures are marked in seconds. The results are displayed in Fig. 8 (rights panel). These data suggest that the saturation coverage of S-2-butanol is larger than that of R-2-butanol so that NEA provides an enantioselective surface. The ER ratio obtained from:

$$
E R_{1-(1-n a p h t h y l)-e \text { thyla mine }}=\frac{\theta_{S-2-b u \text { tanol }}}{\theta_{R-2-b u \text { tanol }}}
$$

is displayed in the right panel of Fig. 6.
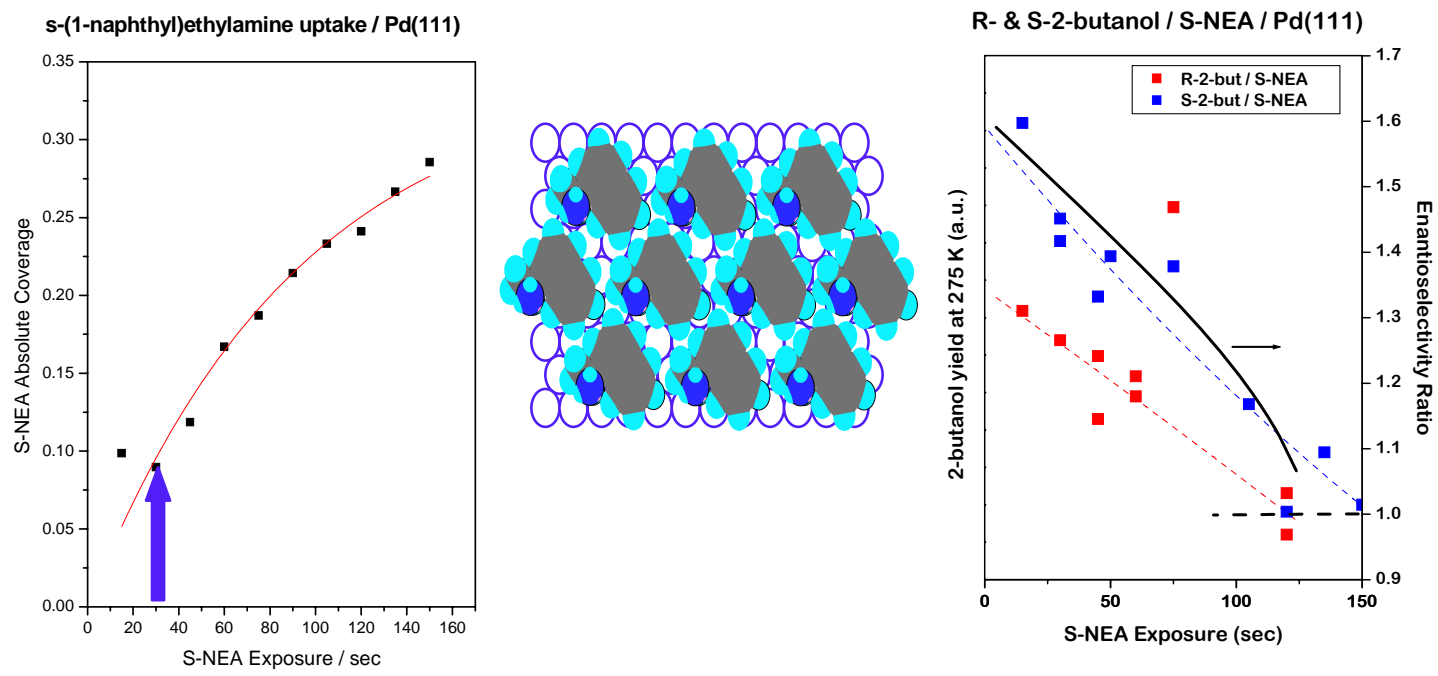

Figure 8: Uptake of S-1-(1-naphthyl)-ethylamine on $\operatorname{Pd}(111)$ as a function of exposure measured by XPS(left panel), and a desorption of a monolayer of S-1-(1-naphthyl)-ethylamine and the adsorption of R- and S-2-butanol on S-1-(1-naphthyl)-ethylamine-covered $\operatorname{Pd}(111)$ (right panel).

\section{References}

1. I. Lee and F. Zaera, Enantioselectivity of Adsorption Sites Created by Chiral 2butanol Adsorption on Pt(111) Single-crystal Surfaces, J. Phys. Chem. B., 109, 12920 (2005) 
2. D. Stacchiola, L. Burkholder and W.T. Tysoe, Enantioselective Chemisorption on a Chirally Patterned Surface in Ultrahigh Vacuum: Adsorption of Propylene Oxide on 2-butoxy-Covered Pd(111), J. Am. Chem. Soc., 124, 8984 (2002)

3. Darío Stacchiola, Luke Burkholder and Wilfred T. Tysoe, Enantioselective Chemisorption on a Chirally Modified Surface in Ultrahigh Vacuum: Adsorption of Propylene Oxide on 2-butoxide-Covered Pd(111), J. Mol. Catal A: Chemical, 216, 215 (2004)

4. F. Roma, D. Stacchiola, G. Zgrablich and W. T. Tysoe, Theoretical Analysis of the Coverage Dependence of Enantioselective Chemisorption on a Chirally Patterned Surface, Journal of Chemical Physics, 118, 6030 (2003)

5. F. Romá, D. Stacchiola, W.T. Tysoe and G. Zgrablich, Lattice-gas Modeling of Enantioselective Adsorption by Template Chiral Substrates, Physica A., 338, 493 (2004)

6. H. C. Poon, M. Weinert, D. K. Saldin, D. Stacchiola, T. Zheng and W. T. Tysoe, Structure Determination of Disordered Organic Molecules on Surfaces from the Bragg Spots of Low Energy Electron Diffraction and Total Energy Calculations, Phys. Rev. B., 69, 35401 (2004)

7. M. Bonello, F.J. Williams and R.M. Lambert, Aspects of Enantioselective Heterogeneous catalysis: Structure and Reactivity of S-1-(1-naphthyl)-ethylamine on Pt $\{111\}$, J. Am. Chem. Soc., 125, 2723 (2003)

8. Chemistry of Glycine on Pd(111): Temperature-programmed Desorption and Xray Photoelectron Spectroscopic Study, Feng Gao, Zhenjun Li, Yilin Wang, Luke Burkholder and W.T. Tysoe, J. Phys. Chem. C., submitted

9. Chemistry of Alanine on $\operatorname{Pd}(111)$ : Temperature-programmed Desorption and Xray Photoelectron Spectroscopic Study, Feng Gao, Zhenjun Li, Yilin Wang, Luke Burkholder and W.T. Tysoe, Surf. Sci. submitted

\section{List of Publications Acknowledging DOE Support}

Theoretical Analysis of the Coverage Dependence of Enantioselective Chemisorption on a Chirally Patterned Surface, F. Roma, D. Stacchiola, G. Zgrablich and W. T. Tysoe, Journal of Chemical Physics, 118, 6030 (2003)

Probing Enantioselective Chemisorption in Ultrahigh Vacuum, Darío Stacchiola, Luke Burkholder and Wilfred T. Tysoe, J. Mol. Catal A: Chemical, 216, 215 (2004)

Lattice-gas Modeling of Enantioselective Adsorption by Template Chiral Substrates, F. Romá, D. Stacchiola, W.T. Tysoe and G. Zgrablich, Physica A., 338, 493 (2004)

Requirements for the Formation of a Chiral Template, D. Stacchiola, L. Burkholder, T. Zheng, M. Weinert and W.T. Tysoe, J. Phys. Chem. B.,109, 851 (2005)

The Structure of Formate Species on Pd(111) Calculated by Density Functional Theory and Determined Using Low Energy Electron Diffraction, T. Zheng, D. Stacchiola, D.K. Saldin, J. James, D.S. Sholl and W.T. Tysoe, Surface Science, 574, 166 (2005) 
Structure and Binding Site of Acetate on Pd(111) Determined Using Density Functional Theory and Low Energy Electron Diffraction, Joanna James, Dilano K. Saldin, T. Zheng, W. T. Tysoe and David S. Sholl, Catalysis Today, 105, 74 (2005)

Chemistry of Glycine on Pd(111): Temperature-programmed Desorption and X-ray Photoelectron Spectroscopic Study, Feng Gao, Zhenjun Li, Yilin Wang, Luke Burkholder and W.T. Tysoe, J. Phys. Chem. C., submitted

Study of Thermal Programmed Desorption of Chiral Propylene-Oxide from Pd(111) Surfaces, V. Bustos, W.T. Tysoe, Feng Gao, Yilin Wang, Luke Burkholder and G. Zgrablich, in preparation

Chemistry of Glycine on $\mathrm{Pd}(111)$ : Temperature-programmed Desorption and X-ray Photoelectron Spectroscopic Study, Feng Gao, Zhenjun Li, Yilin Wang, Luke Burkholder and W.T. Tysoe, Surf. Sci., submitted

\section{Financial Statement}

No unobligated funds are anticipated at the end of the funding period. 